\title{
Editorial
}

\section{Safe, Resilient, and Sustainable Transportation Systems}

\author{
Yuanchang Xie, ${ }^{1}$ Pan Liu, ${ }^{2}$ and Chronis Stamatiadis ${ }^{1}$ \\ ${ }^{1}$ Civil and Environmental Engineering, University of Massachusetts Lowell, 1 University Avenue, Lowell, MA 01854, USA \\ ${ }^{2}$ School of Transportation, Southeast University, 2 Sipailou, Nanjing, Jiangsu 210096, China \\ Correspondence should be addressed to Yuanchang Xie; yuanchang_xie@uml.edu
}

Received 2 December 2015; Accepted 2 December 2015

Copyright (c) 2016 Yuanchang Xie et al. This is an open access article distributed under the Creative Commons Attribution License, which permits unrestricted use, distribution, and reproduction in any medium, provided the original work is properly cited.

Traffic accidents are among the top 10 causes of death worldwide. They resulted in over 1.3 million fatalities in 2012 alone and cause billions of dollars of loss each year in productivity, property damage, and time and fuel due to congestion. Traffic safety is becoming increasingly important, particularly in developing countries that are facing rapid urbanization and motorization. In addition to traffic safety, there are many recent examples of transportation systems being disrupted by extreme weather events. Transportation activities, on the other hand, also contributed substantially to these extreme weather events either directly or indirectly. Clearly, it is important to build a resilient and sustainable transportation system that is less vulnerable to disasters and has the minimum possible environmental footprint.

In this special issue, several studies investigated highway transportation from the operations, resiliency, and logistics perspectives. X. Zhang et al. developed a cooperative Qlearning algorithm for finding near-optimal path sets for multiple Origin-Destination (OD) pairs simultaneously. The new algorithm was compared with the $k$-shortest path algorithm and the Dijkstra's algorithm. The comparison result shows that the new algorithm can generate more reliable shortest path solutions that take into consideration failures of road segments and intersections due to congestion or traffic accidents. L. Yang et al. modified the desired minimum gap and structure of the Intelligent-Driver Model (IDM) to better account for the real-time maximum deceleration. They applied the new IDM to Adaptive Cruise Control (ACC) and simulated it under two road conditions. They found that the new model can improve the safety and stability of ACC. It can also increase roadway capacity. Accurate queue length estimation can be very useful in developing effective traffic control strategies to improve mobility and reduce traffic emissions. S Zhao et al. modified the Cell Transmission Model (CTM) for signalized intersection queue length estimation. The new CTM was tested based on the queue length data simulated by a calibrated VISSIM model. It was found to perform well under both undersaturated and saturated traffic conditions and can accurately model both queue forming and dissipating processes. Accurate traffic flow predictions are also important to traffic signal control. A. O. Adewumi et al. proposed a traffic flow prediction model based on Chaos Theory by computing the largest Lyapunov Exponent. To model how intersection and road segment failures propagate in a traffic network, Y. Zhang et al. proposed a Coupled Map Lattice (CML) method, based on which simulations were conducted to evaluate the impacts of various factors on network failures. As an essential component of autonomous/selfdriving vehicles, Traffic Sign Detection and Recognition (TSDR) systems are very important for traffic safety. S. B. Wali et al. developed a fast automatic TSDR system based on RGB color segmentation, shape matching, and a support vector machine classifier. The developed system generated promising detection and false positive rates under various lighting and viewing angle conditions. J. A. Marmolejo et al. studied the design of capacity-constrained distribution networks to minimize fixed and transportation costs. They formulated the problem as a mixed-integer program and proposed a Benders decomposition algorithm to solve it. The Benders decomposition algorithm was compared with CPLEX. The results suggest that it can closely approximate the optimal solutions generated by CPLEX with significantly less computation time and is suitable for solving large-scale distribution network design problems. C. Wu et al. developed 
a Transportation Supply-Demand Ratio (TSDR) model to quantitatively assess whether an urban area's transportation supply can meet its demand for sustainable development. This TSDR model is based on the system dynamic principle and was evaluated using VENSIM simulation.

Given the rapid development of high-speed rail in China, the aging rail infrastructure in developed countries, and many high-profile rail accidents, railroad safety has been receiving increasing attention globally. P. Xu et al. developed a railroad track condition prediction model and demonstrated its accuracy and robustness using track geometry data. L. Song et al. focused on analyzing the safety and reliability of high-speed Electric Multiple-Unit (EMU) trains. They integrated Fault Tree Analysis (FTA) and Petri nets and demonstrated their effectiveness in analyzing the failure modes of pantograph of high-speed EMU trains. J. Ye et al. proposed an improved Optimal Velocity (OV) car-following model for moving-block train control. Compared to the conventional OV car-following model, the improved $\mathrm{OV}$ model takes into consideration uncertainties in distance headway measurements and generates safer and more stable train control performance.

Extreme weather events have caused many airline flight disruptions. B. Zhu et al. developed a two-stage stochastic model to optimize the recovery process of airline flight disruptions. The first stage adjusts flight schedules to minimize delay and flight cancellation cost. The second stage focuses on minimizing aircraft rerouting cost. The authors also developed a simulated annealing algorithm to solve the two-stage model. G. Duan et al. focused on aircraft crash modeling. They improved the Functional Resonance Analysis Method (FRAM) by integrating it with model checking, which allows researchers to use the FRAM for identifying and simulating all possible ways that a hazard factor may lead to accidents. They demonstrated the enhanced FRAM's effectiveness by applying it to analyze a two-aircraft crash in Italy.

Public transit plays a major role in battling traffic emissions and global warming. To make public transit more attractive, it is important to have well-developed transit assignment models for optimal transit network design. S. Luo et al. investigated the independence between the travel times of adjacent transit links, which has been implicitly assumed to be independent in most transit assignment methods. They developed a method to determine the validity of the travel time independence assumption. They also proposed a capacity-restraint assignment method to handle the case where the travel time independence assumption is invalid.

This special issue includes a broad range of topics related to safe, resilient, and sustainable transportation systems. We received 47 manuscripts and 14 of them were accepted for publication, covering highway, rail, air, and transit modes.

\section{Acknowledgments}

We would like to sincerely thank all authors who contributed their papers to this special issue. Also, we are extremely grateful to all reviewers for their time and outstanding work, which are critical to the success and quality of this special issue.

Yuanchang Xie

Pan Liu

Chronis Stamatiadis 


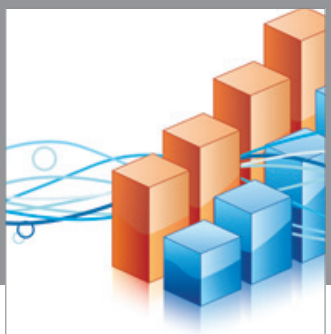

Advances in

Operations Research

vatem alat4

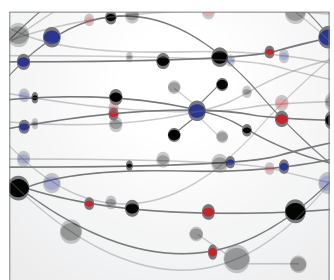

\section{The Scientific} World Journal
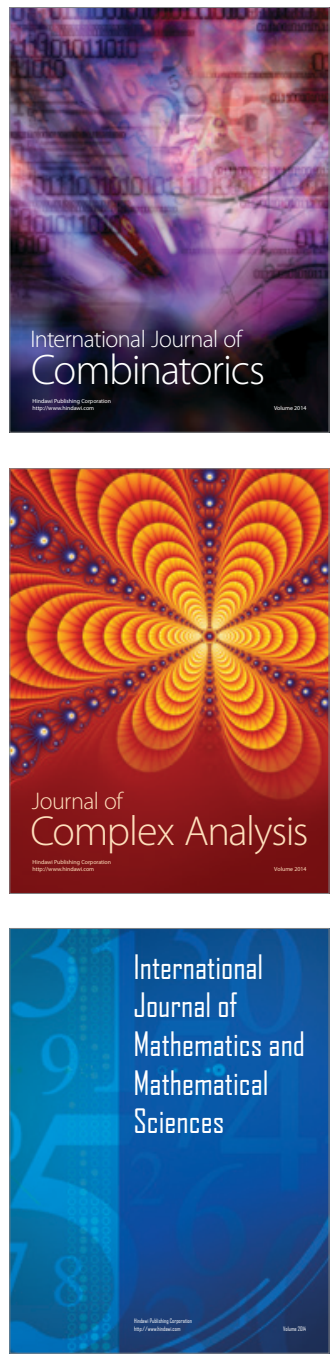
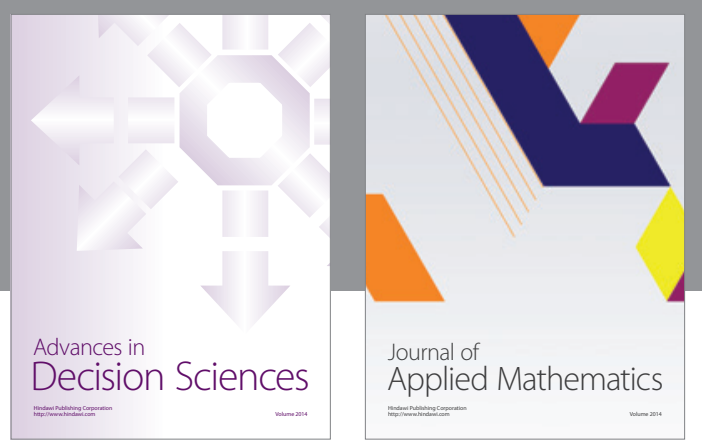

Algebra

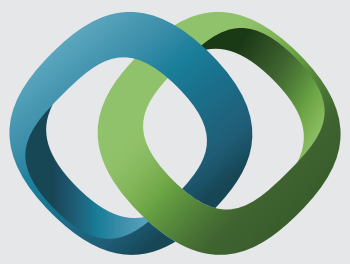

\section{Hindawi}

Submit your manuscripts at

http://www.hindawi.com
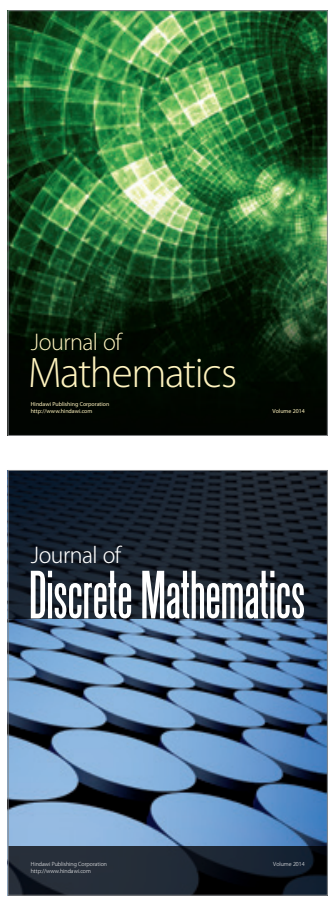

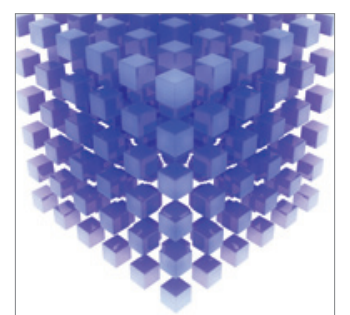

Mathematical Problems in Engineering
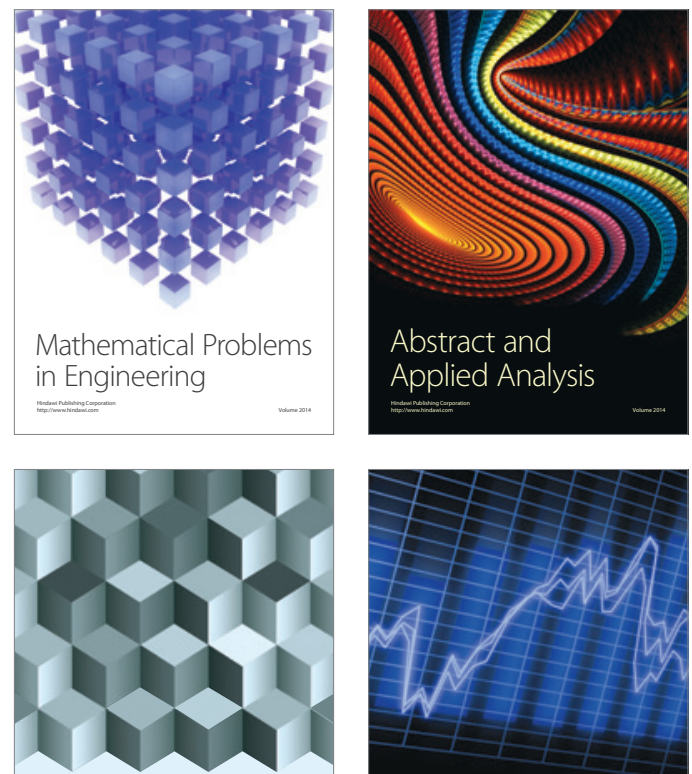

Journal of

Function Spaces

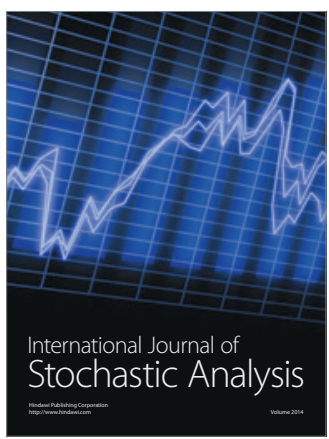

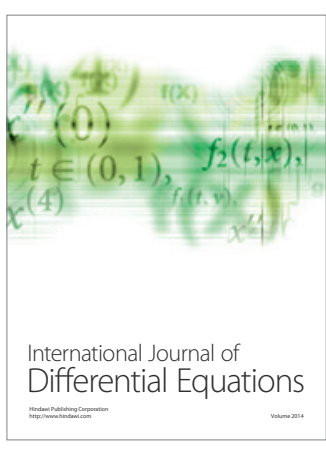
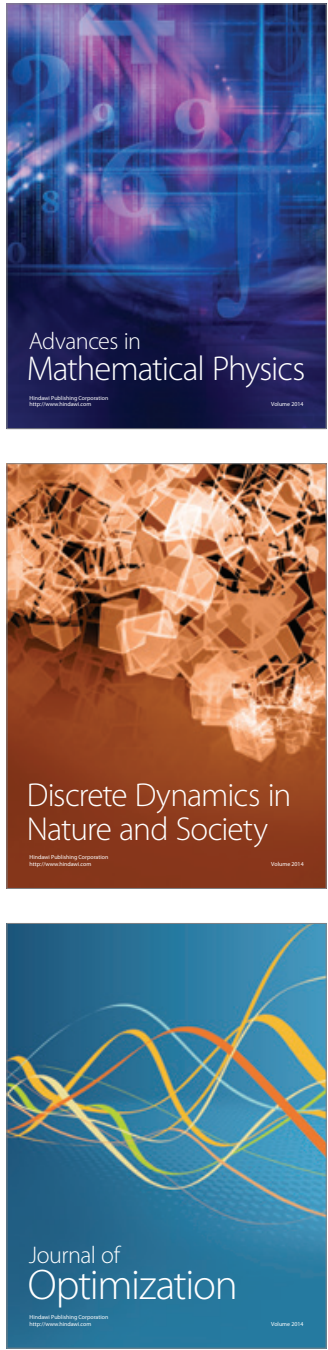